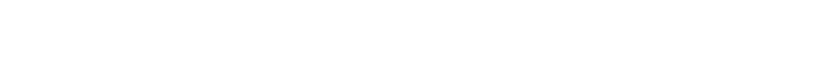

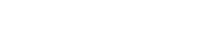

Łŷrúc ũ ũté

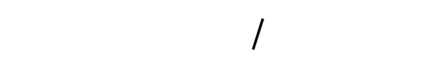

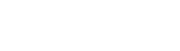

rŮt ŤKE

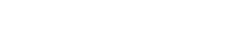

ū ũté

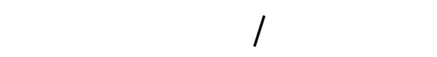

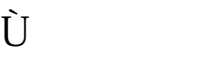

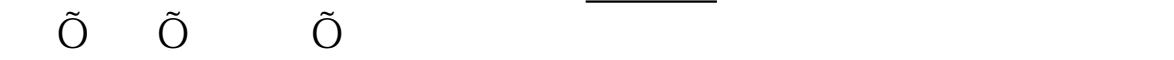

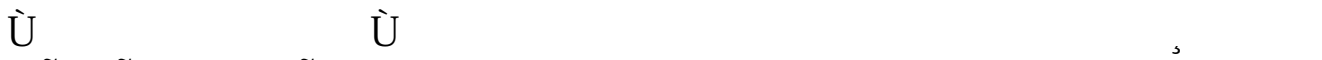

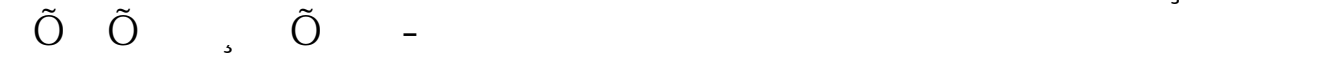

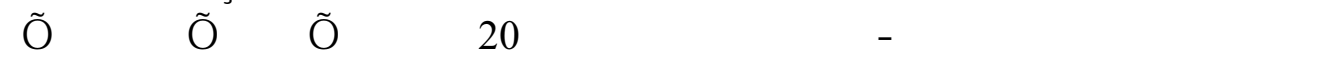

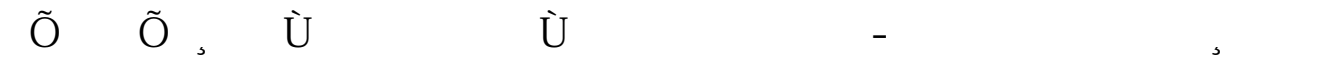

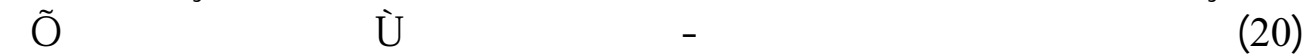

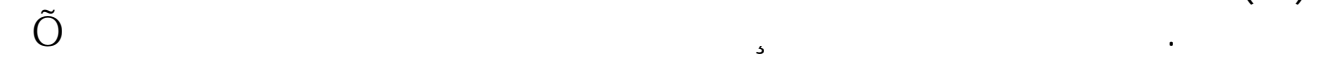
r̀»

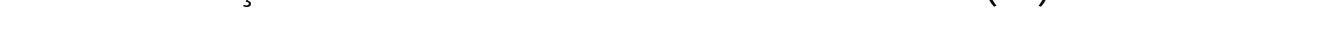
Doorenbos ) rॅ»

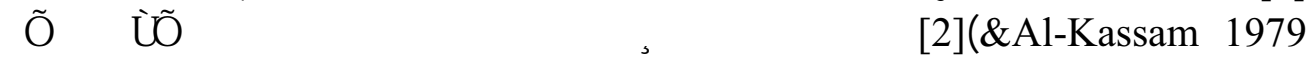

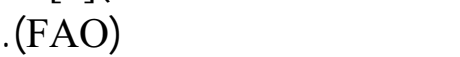

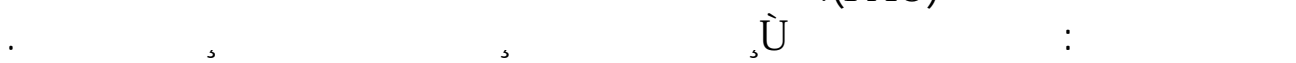

\section{Predicting Yield Response Factors In Jensen Model}

Eman Hazim Sheet

University of Mosul

College of Engineering

Water Resources Dept.
Maan Hazim Sheet

Univresity of Dihouk

College of Engineering

Water Resources Dept.

\section{$\underline{\underline{\text { Ebstract }}}$}

The yield response model is very important in irrigation planning and management of irrigation projects. A computerized model for simulating irrigation water requirement for wheat crop at Mosul area has been proposed for (20)years of daily climatological data. The model predict daily actual and potential evapotranspiration, then the relation between relative yield and relative evapotranspiration is put into (20)equations and solved mathematically to find the coefficients for each stage of growing growth period of wheat crop at Mosul area.The yield response factors is found for two cases, the first is for rainfed agriculture practice for wheat for (20)years where an available data for production is available , and the second is for irrigated practice where four years production data were available[1] .Then the results are compared with the results obtained from (Doorenbos \& Al kassam 1979) equation for yield. [2] and show a small variation while the last equation is justified by International Agriculture and Food Organization(FAO).

Keywords: crop water production function ,yield response factor, yield Jensen model, irrigation management. 


\section{retose}

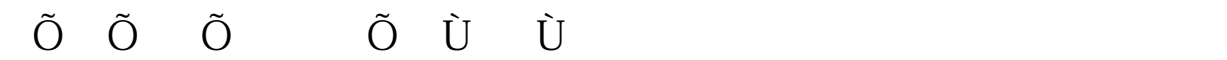

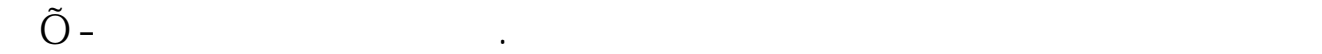

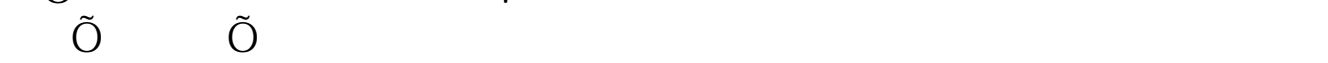

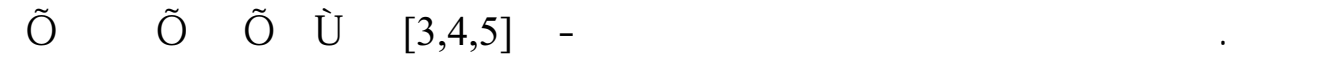

$\mathrm{Y} / \mathrm{Y}_{\max }=\mathrm{T} / \mathrm{T}_{\max }$

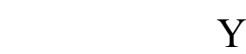

:EŔŜ\&

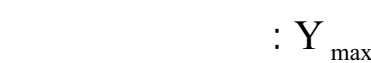

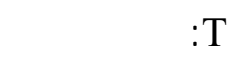

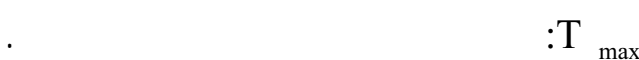

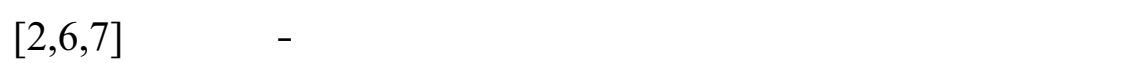

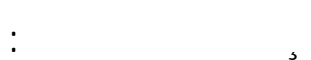

$$
\mathrm{Y} / \mathrm{Y}_{\max }=\prod_{i=1}^{n}\left(\mathrm{AET}_{i} / \mathrm{CPET}_{i}\right)^{\lambda_{i}}
$$

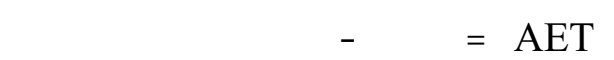

:EŔŝষ

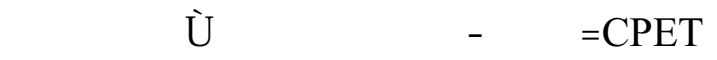

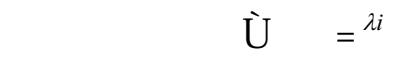

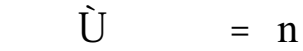

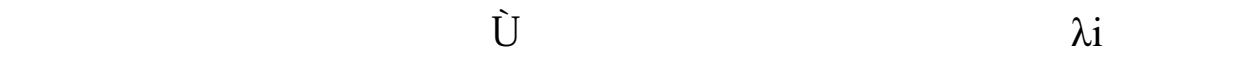

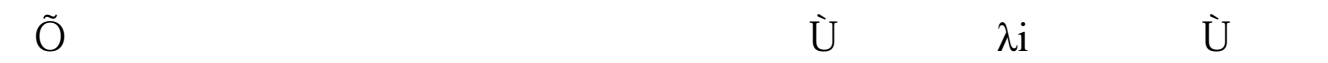

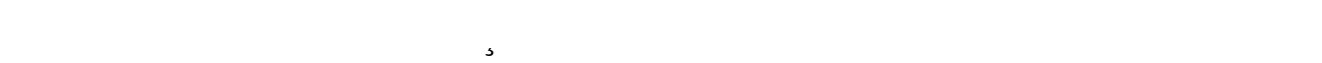

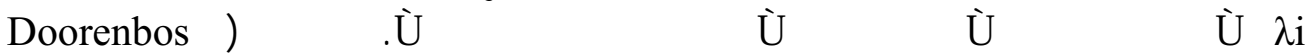

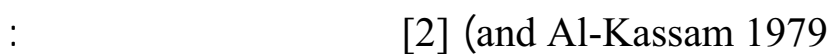

$$
\mathrm{Y} / \mathrm{Ymax}=\prod_{i=1}^{n}\left[1-\mathrm{ky}_{i}\left(1-\mathrm{AET}_{i} / \mathrm{CPET}_{i}\right)\right]
$$




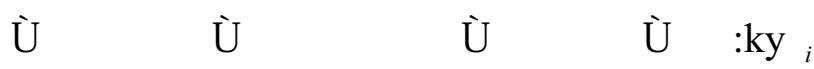

:EŔŝষ

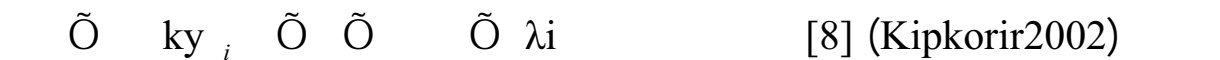

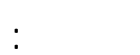

$$
\lambda=0.275 \mathrm{ky}^{3}-0.1351 \mathrm{ky}^{2}+0.8761 \mathrm{ky}-0.0187
$$

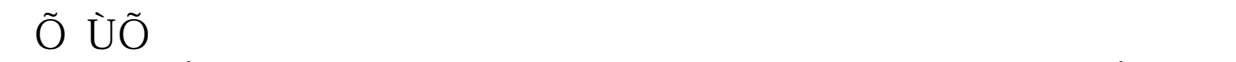

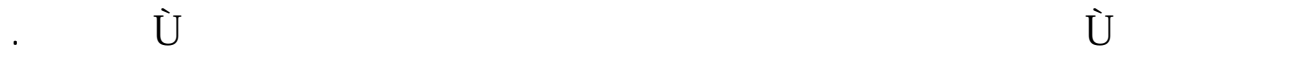

\section{Śšititìģžłü}

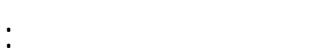

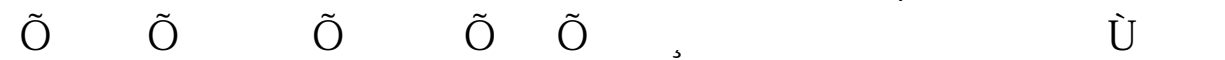

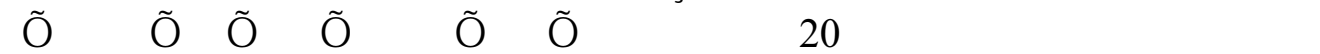

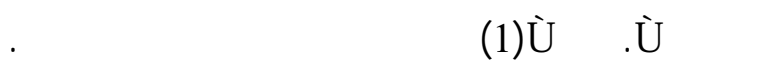
G) Ž

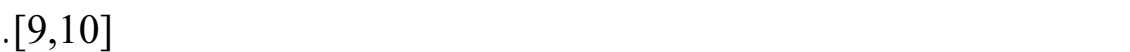

$$
E T_{\circ}=\frac{0.408 \Delta\left(R_{n}-G\right)+\gamma \frac{900}{T+273} U_{2}(e s-e a)}{\Delta+\gamma\left(1+0.34 U_{2}\right)} \ldots \ldots(5)
$$

$\mathrm{mm} / \mathrm{day}$

${ }^{\circ} \mathrm{c}$

$\mathrm{m} / \mathrm{sec}$

$\mathrm{MJ} / \mathrm{m}^{2}$ day

$\mathrm{MJ} / \mathrm{m}^{2}$ day

$\mathrm{KPa}$

$\mathrm{KPa}$

$\mathrm{KPa}$

$\mathrm{KPa} / \mathrm{c}^{\circ}$

$\mathrm{KPa} / \mathrm{c}^{\circ}$

:ĐŔŝষ

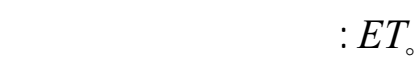

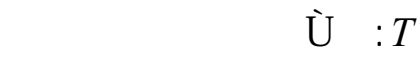

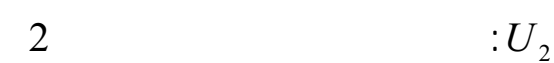

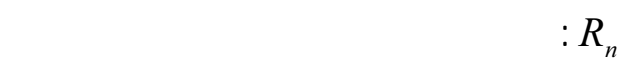

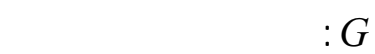

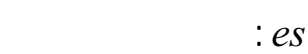

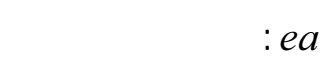

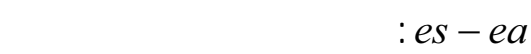

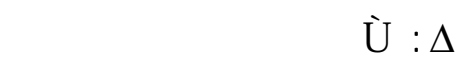

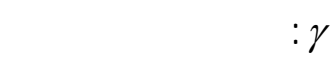




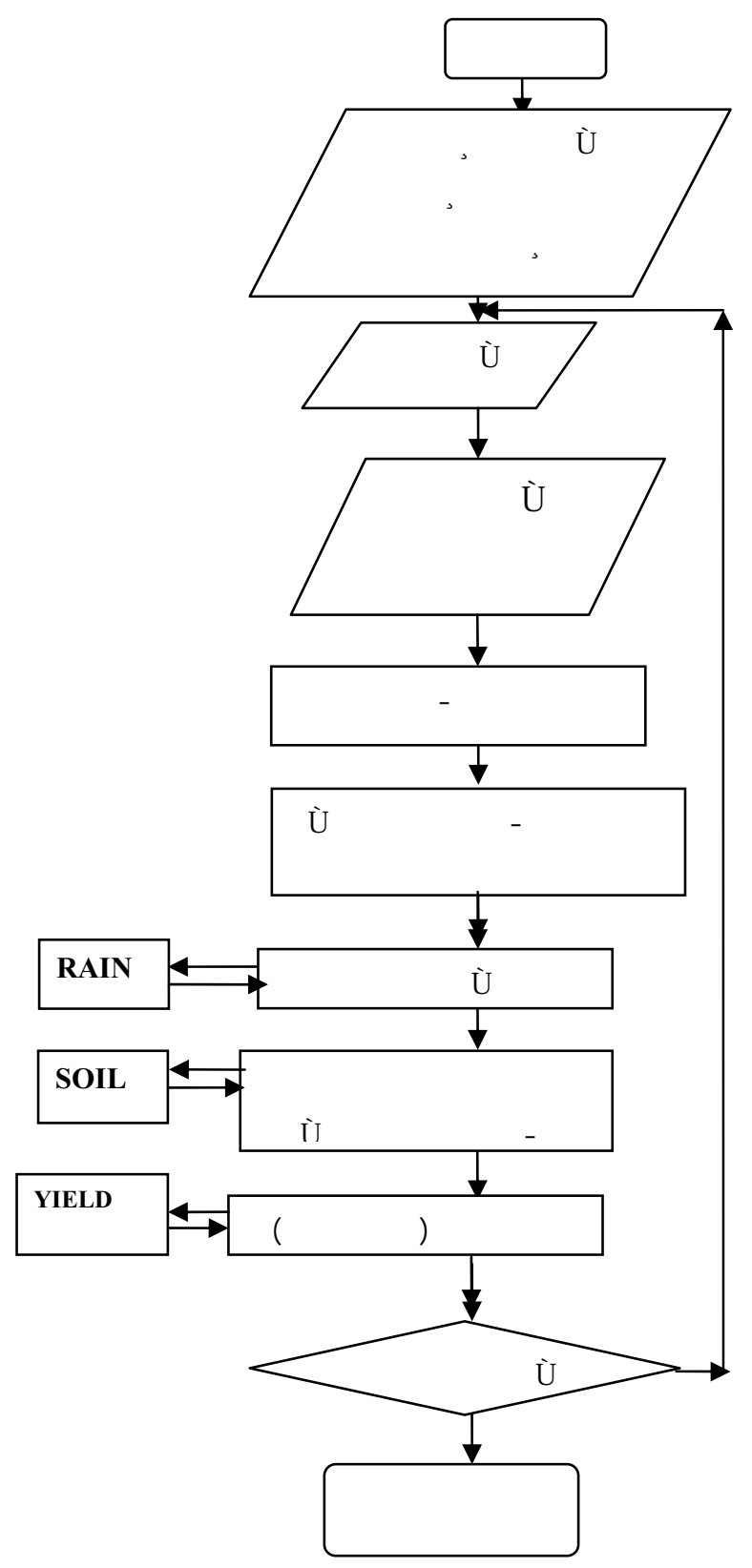

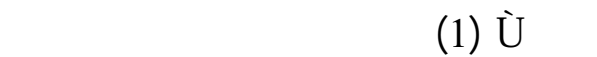




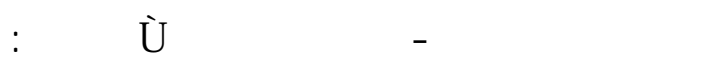

$\mathrm{CPET}=\mathrm{Kc} *$ Eto

$$
\begin{aligned}
& \mathrm{mm}
\end{aligned}
$$

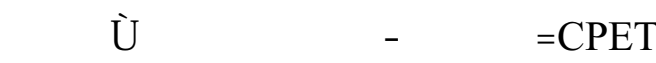

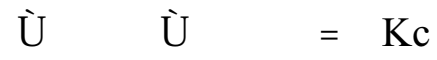

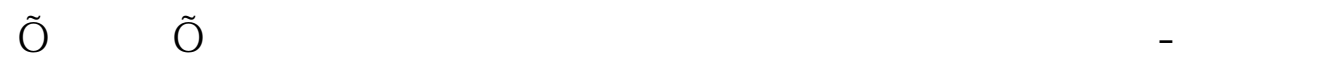

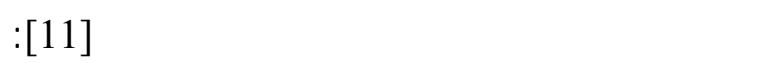

$$
\mathrm{AET}=\mathrm{CPET} * \mathrm{SK}
$$

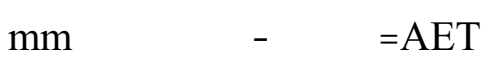

$$
\text { :EŔŜ\& }
$$

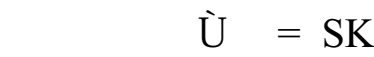

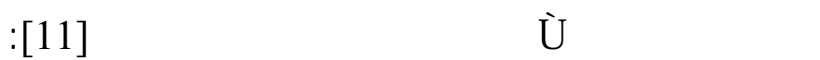

$$
\mathrm{SK}=\log (1+\mathrm{PAW}) / \log 101
$$

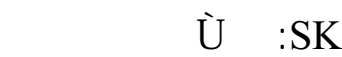

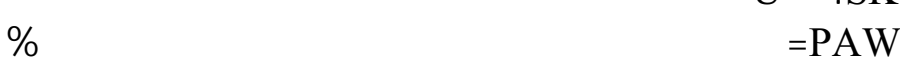

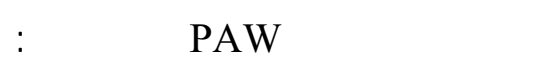

$$
\mathrm{PAW}=(\mathrm{AW} / \mathrm{TAW}) * 100 .
$$

$$
\begin{aligned}
& \text { :EŔŝళ } \\
& \mathrm{mm}
\end{aligned}
$$

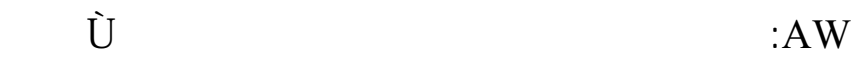

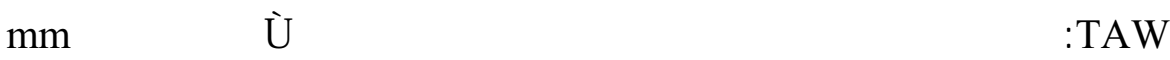

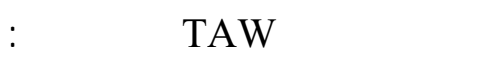

$$
\begin{aligned}
& \text { TAW=RZ*(FC-PWP) }
\end{aligned}
$$




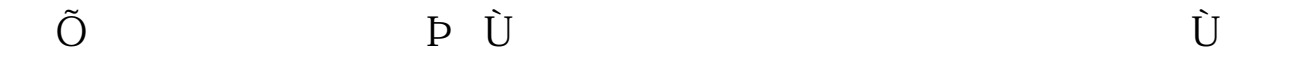
'mí dŔk

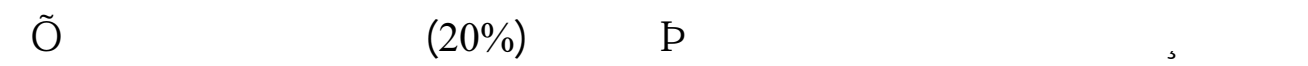

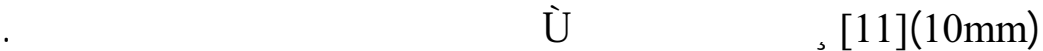

$\mathrm{AWi}+\mathrm{IRR}+\mathrm{RAIN}=\mathrm{AET}+\mathrm{DRAIN}+\mathrm{AWe}$

$\mathrm{mm}$

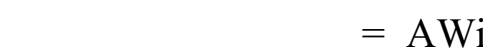

$\mathrm{mm}$

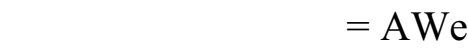

$\mathrm{mm}$

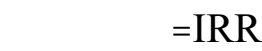

$\mathrm{mm}$ ũudR'́n'́c =RAIN $\mathrm{mm}$ ¿ŪHŔ'níc =DRAIN

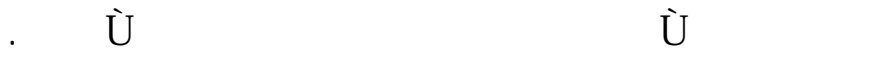

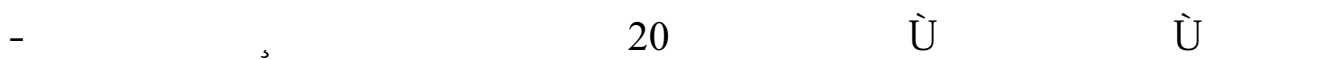

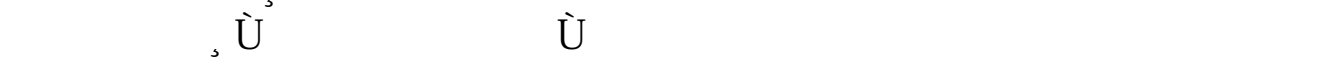

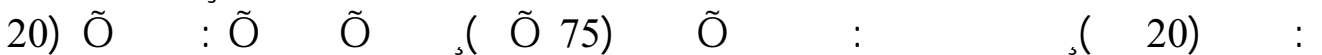

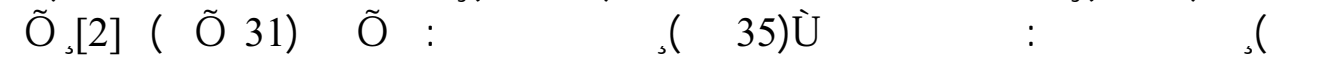

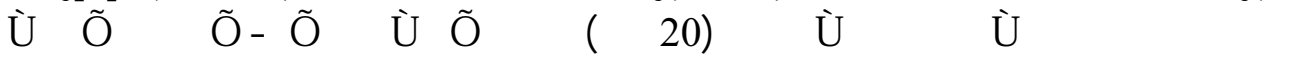

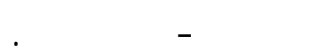

F»

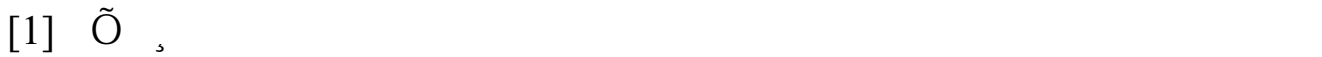

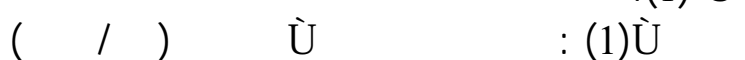

\begin{tabular}{|c|c|c|}
\hline \multicolumn{2}{|c|}{ 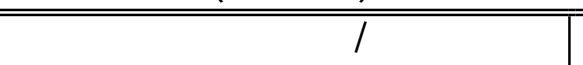 } & \multirow[b]{2}{*}{ Řáḱtîg } \\
\hline $\begin{array}{r}\text { G»» Plbśf ũ } \\
\text { ¿ớb }\end{array}$ & ù Bícifbśf ũ & \\
\hline 2930 & 2760 & $95 / 11 / 29$ \\
\hline 4081 & 4046 & $96 / 1 / 2$ \\
\hline 3952 & 3872 & $96 / 2 / 7$ \\
\hline 5500 & 4710 & $96 / 12 / 1$ \\
\hline 5470 & 4560 & $97 / 1 / 5$ \\
\hline 3490 & 3130 & 97/2/1 \\
\hline 4610 & 2160 & $97 / 12 / 30$ \\
\hline 1090 & 460 & 97/2/17 \\
\hline
\end{tabular}




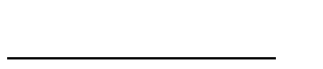

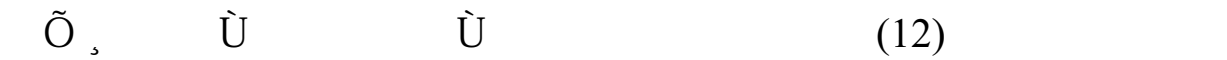

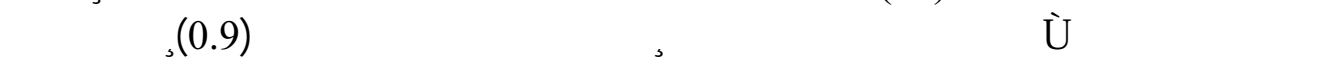

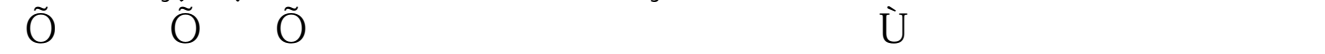

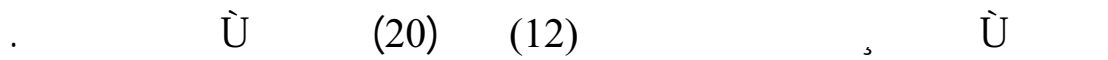
$0.90=(0.75)^{\lambda 1}(0.91)^{\lambda 2}(0.96)^{\lambda 3}(0.89)^{\lambda 4}(0.91)^{\lambda 5}$ $0.99=(0.55)^{\lambda 1}(0.90)^{\lambda 2}(0.96)^{\lambda 3}(0.91)^{\lambda 4}(0.97)^{\lambda 5}$ $1.00=(0.55)^{\lambda 1}(0.90)^{\lambda 2}(0.96)^{\lambda 3}(0.89)^{\lambda 4}(0.91)^{\lambda 5}$ $0.97=(0.86)^{\lambda 1}(0.90)^{\lambda 2}(0.47)^{\lambda 3}(0.65)^{\lambda 4}(0.89)^{\lambda 5}$

$0.95=(0.86)^{\lambda 1}(0.90)^{\lambda 2}(0.47)^{\lambda 3}(0.80)^{\lambda 4}(0.90)^{\lambda 5}$

$0.97=(0.90)^{\lambda 1}(0.90)^{\lambda 2}(0.47)^{\lambda 3}(0.66)^{\lambda 4}(0.25)^{\lambda 5}$

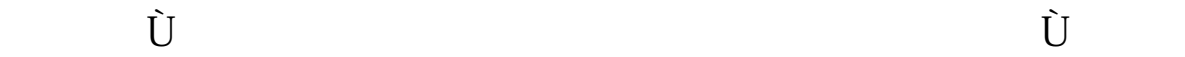

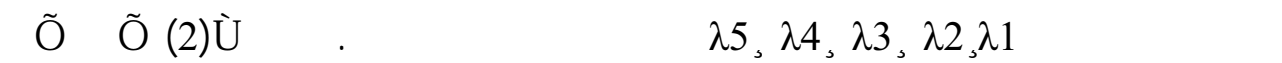

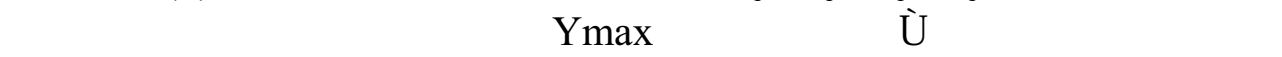

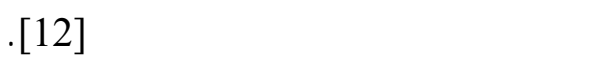

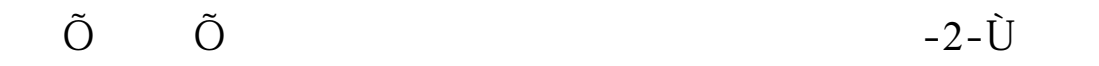

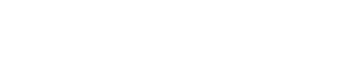

\begin{tabular}{||c|c|c|c|c||}
\hline$\lambda 1$ & $\lambda 2$ & $\lambda 3$ & $\lambda 4$ & $\lambda 5$ \\
\hline 0.76 & 3.14 & 0.32 & 3.84 & 2.74 \\
\hline
\end{tabular}




\begin{tabular}{llll} 
Al-Rafidain Engineering & Vol.14 & No.3 & 2006 \\
\hline
\end{tabular}

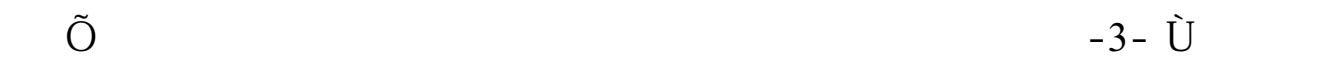

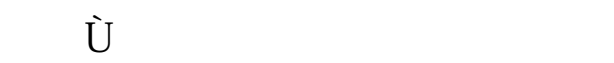

\begin{tabular}{|l|r|r|r|r||}
\hline$\lambda 1$ & $\lambda 2$ & $\lambda 3$ & $\lambda 4$ & $\lambda 5$ \\
\hline 0.41 & 3.07 & 0.01 & -4.58 & 3.41 \\
\hline
\end{tabular}

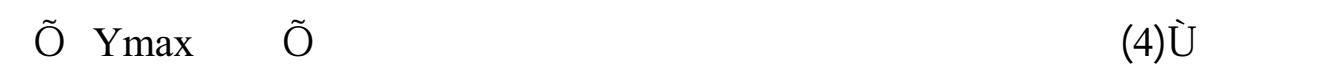

.ry

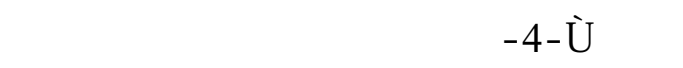

\begin{tabular}{|c|c|c|c|c|}
\hline$\frac{12 \lambda 1}{21}$ & $\overline{\lambda 22}$ & $\overline{l \lambda 3}$ & $\overline{\overline{\lambda 4}}$ & $\overline{\lambda \bar{~}}$ \\
\hline 0.54 & -20.2 & 8.0 & 2.98 & 0.37 \\
\hline
\end{tabular}

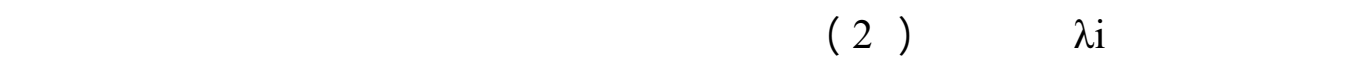

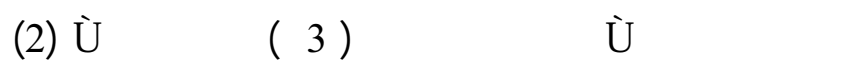

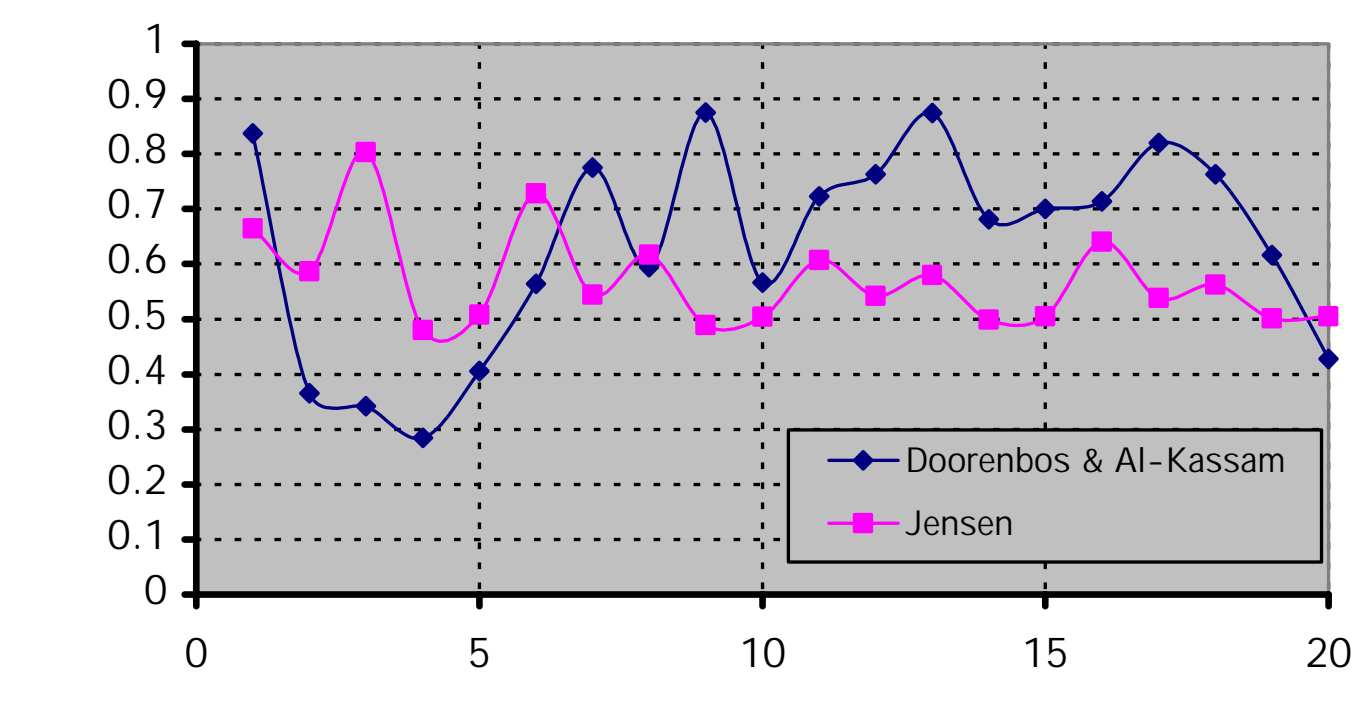

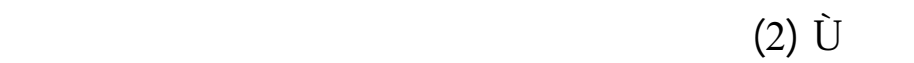




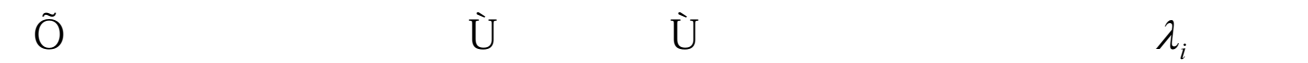

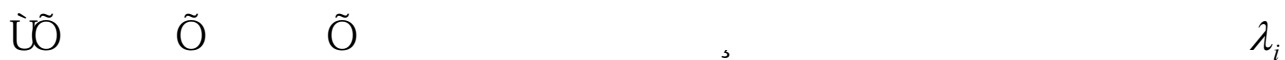

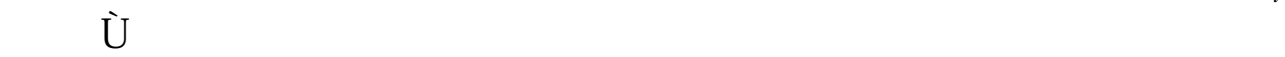

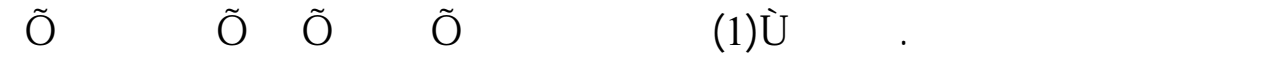

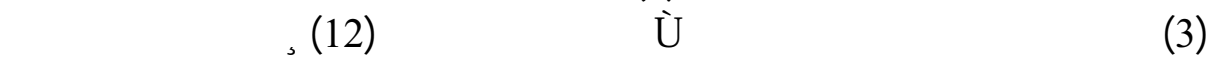

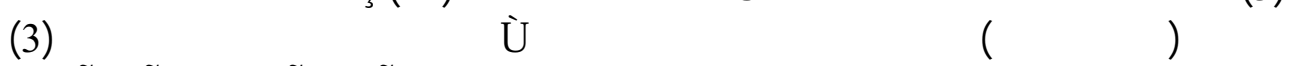

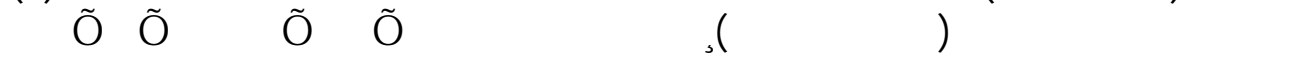

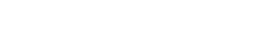

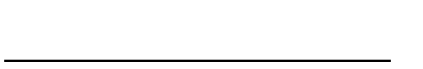

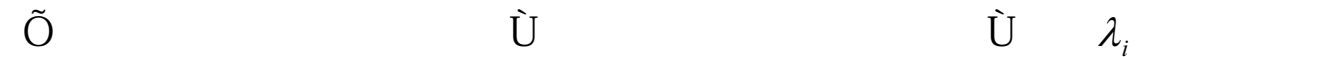

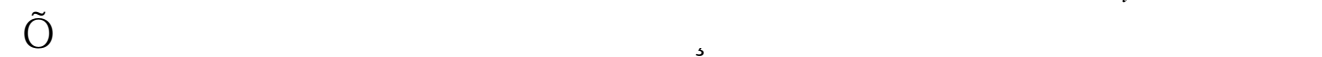

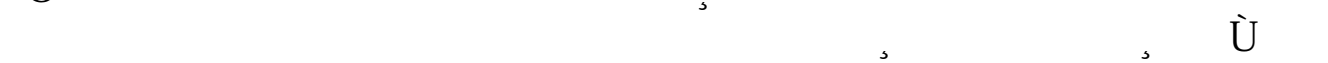

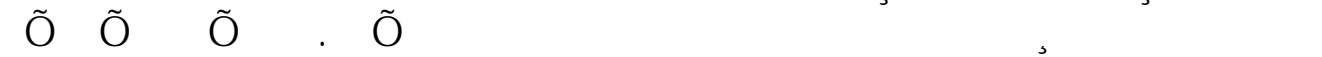

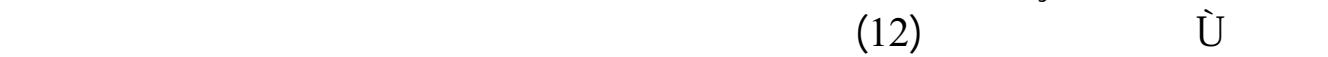
tbö

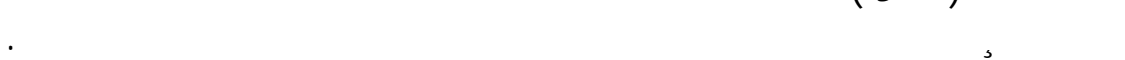

\section{tetderie}

(1)Adary et al. "Wheat productivity under supplemental irrigation in northern Iraq" ,ICARDA,2002.

(2)Doorenbos J. \& Kassam A.H. "Yield response to water".FAO,Irrigation \& Drainage paper No.33,Rome,Italy,1979,p.193.

(3)Hiler E.A. \& Clark R.N. "Stress day index to characterize effects of water stress on crop yield". Transactions of the ASAE 14,1971,p.757-761.

(4)Stewart J.I. \& Hagan R.M. "Function to predict the effects of crop water deficit".Journal of the irrigation and drainage divisions,ASCE 99,1973,p.421-439.

(5)Hanks R.J."Model for predicting plant yield as influenced by water use".Agronomy journal 66,1974,p.660-665.

(6)Howell,T.A. \& Hiler E.A. "Optimization of water use efficiency under high frequency irrigation evapotranspiration \& yield relationship,Transactions of the ASAE,99,1975,p.873-878.

(7)Jensen M.E. "Water consumption by agriculture plants,ch.1 in:Kozlowski,Water deficit and plant growth vol II,Academic press New York,1968,p.1-22.

(8)Kipkorir E.C. \&Raes D. "Transformation of yield response factor into Jensen sensitivity index". Irrigation \& Drainage system,16,2002,p.47-52 
(9)Richard G.A. et al.."Crop Evapotranspiration guidelines for computing crop water requirements" FAO,Irrigation \& Drainage paper No.56,Rome,Italy, 1998.

(9)Richard G.A. et al.."Crop Evapotranspiration guidelines for computing crop water requirements" FAO,Irrigation \& Drainage paper No.56,Rome,Italy, 1998.

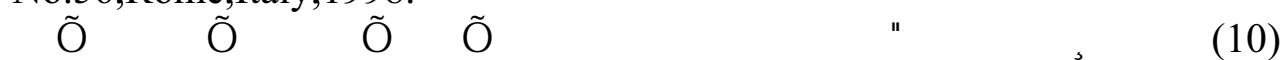

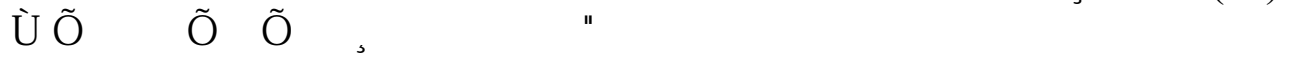

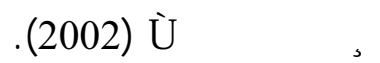

(11)Sheet,E.H.(1987)."Modelling supplemental irrigation water requirements for wheat crop at Mosul area". M.SC.thesis submitted to the college of Engineering,University of Mosul.

(12)Zhang Z. \& Pei D. et al "Management of supplemental irrigation of winter wheat for max. profit". http://www.fao.org/Docrep/004/y3655e09.htm,2004.

13)Chahraman,B \& Sepaskha A.R. "Use of water deficit \& sensitivity index for partial irrigation scheduling of Wheat \&Barely".Irrigation science, 18,1997,p.11-16. 